\title{
The emergency to home project: impact of an emergency department care coordinator on hospital admission and emergency department utilization among seniors
}

\author{
Christopher Matthew Bond ${ }^{1 *}$, Elizabeth A Freiheit ${ }^{2}$, Lesley Podruzny ${ }^{3}$, Alianu Akawakun Kingsly², Dongmei Wang ${ }^{1}$, \\ Jamie Davenport ${ }^{3}$, Abram Gutscher ${ }^{3}$, Cathy Askin ${ }^{4}$, Allison Taylor ${ }^{3}$, Vivian Lee ${ }^{3}$, Queenie Choo ${ }^{3}$ and Eddy Samuel Lang
}

\begin{abstract}
Background: Seniors comprise $14 \%$ to $21 \%$ of all emergency department (ED) visits, yet are disproportionately larger users of ED and inpatient resources. ED care coordinators (EDCCs) target seniors at risk for functional decline and connect them to home care and other community services in hopes of avoiding hospitalization.

The goal of this study was to measure the association between the presence of EDCCs and admission rates for seniors aged $\geq 65$. Secondary outcomes included length of stay, recidivism at 30 days, and revisit resulting in admission at 30 days.

Methods: This was a matched pairs study using administrative data from eight EDs in six Alberta cities. Four of these hospitals were intervention sites, in which patients were seen by an EDCC, while the other four sites had no EDCC presence. All seniors aged $\geq 65$ with a discharge diagnosis of fall or musculoskeletal pathology were included. Cases were matched by CTAS category, age, gender, mode of arrival, and home living environment. McNemar's test for matched pairs was used to compare admission and recidivism rates at EDCC and non-EDCC hospitals. A paired t-test was used to compare length of stay between groups.

Results: There were no statistically significant differences for baseline admission rate, revisit rate at 30 days, and readmission rate at 30 days between EDCC and non-EDCC patients.

Conclusions: This study showed no reduction in senior patients' admission rates, recidivism at 30 days, or hospital length of stay when comparing seniors seen by an EDCC with those not seen by an EDCC.
\end{abstract}

Keywords: Geriatric EM care; ED aftercare; Home care; Recidivism; Seniors; Elderly; Falls; Discharge planning

\section{Background}

Compared with younger persons, older adults use emergency services at a higher rate, are more likely to be admitted or have repeat emergency department (ED) visits, and experience higher rates of adverse health outcomes after discharge [1]. In addition to their increased patterns of ED use, frail elderly patients seek care for falls, a presenting complaint not often seen in the young [1]. Furthermore, injuries in the elderly often require more

\footnotetext{
* Correspondence: sperss97@gmail.com

'Department of Emergency Medicine, Foothills Medical Centre, Room C231, 1403-29 St NW, Calgary, Alberta T2N 2 T9, Canada

Full list of author information is available at the end of the article
}

intensive therapy and use disproportionately more resources than would similar injuries in young patients [2]. Several systematic reviews have examined the impact of interventions targeted at improving outcomes for seniors visiting the ED, with mixed results [3,4]. The nature of these interventions has ranged from comprehensive geriatric assessments (CGA) with specially trained ED teams, to telephone follow-up of seniors discharged from the ED [5-7]. Desired outcomes have been variable, and have included quality of life improvement, slowing progression of functional decline, and reducing ED visits and recidivism $[3,4]$. One randomized Australian study showed that seniors' admission rates at 30 days could be reduced through 
assessment by a CGA team prior to their initial ED discharge [8]. Another study showed that a nurse discharge plan coordinator dedicated specifically to the discharge planning care of elderly patients, reduced the proportion of unscheduled ED return visits and facilitated the transition from ED back home and into the community health care network [9].

In order to address the complex needs of seniors visiting the ED, Alberta Health Services ${ }^{\text {a }}$ (AHS), the provincial health authority, commissioned the Emergency to Home Project (E2H) in 2009. E2H connects seniors visiting the ED with services in the community through the efforts of the Emergency Department Care Coordinator (EDCC). The EDCC is a specialized nursing role dedicated to supporting seniors and their caregivers to return home safely after an ED visit. EDCCs educate and advocate for patients and their families by identifying seniors needs outside the ED through conducting an assessment and providing referrals to home care and other community-based services.

In some cases, referral may also involve admission of the patient to an inpatient transition bed until community resources become available. The referrals and supports provided by EDCCs are intended to facilitate meeting seniors' health care needs in the community, thereby reducing repeat ED visits, hospitalization, and hospital length of stay (LOS). Selection of seniors for EDCC consultation was based on the following factors. Seniors who were pre-existing home care clients in the community automatically triggered an EDCC consult when they registered in the ED. If no pre-existing home care was in place, a physician or nurse-initiated consult to the EDCC was required.

The goal of this study was to determine whether the presence of EDCCs would decrease the admission rate of seniors aged $\geq 65$ who have an ED discharge diagnosis of fall or other musculoskeletal (MSK) pathology. We hypothesized that seniors aged $\geq 65$ with falls and MSK injuries are frequently admitted to hospital because of their lack of social supports and the risks associated with ED discharge.

\section{Methods}

\section{Ethics review}

The research question and methods were evaluated using the Alberta Research Ethics Community Consensus Initiative (ARRECI) tool [10]. ARRECI is a four-step, web-based ethics screening tool designed by Alberta Innovates-Health Solutions (formerly the Alberta Heritage Foundation for Medical Research). The project's primary purpose, details of data collected, and risk to participants are inputted into the tool, which then risk stratifies the research project and suggests whether Research Ethics Board approval is needed. Using this tool, it was established that no formal ethics board review was necessary, as the study was low risk and used only anonymous data.

\section{Study design and data sources}

This study used a matched pairs study design using administrative data from eight EDs in six cities in Alberta, Canada. The Data Integration, Measurement and Reporting (DIMR), and Information Management and Technology Services (IMTS) departments of AHS provided the data. Nosologists from IMTS are responsible for coding and abstracting patient diagnoses based on ICD-10 codes and record disposition and LOS data using common tracking systems. Separate information analysts from DIMR subsequently report this data, along with demographic and other information. Seniors presenting with falls and MSK complaints were targeted as they represent a group that is more likely to benefit from ED interventions that arrange a safe discharge plan. The primary outcome was hospital admission rate on index visit. Secondary outcomes included LOS, revisit rate at 30 days, and admission rate at 30 days. In addition to looking at all seniors aged $\geq 65$, cohorts of seniors with identical MSK discharge diagnoses were also analyzed.

\section{Interventions}

Four of the eight hospitals were intervention sites, in which patients were seen by an EDCC, while the remaining four sites were used as a comparator group and had no EDCC presence during the study period of April 1, 2010 to March 31, 2011. The four intervention sites were chosen from EDs with EDCC resources in place, funded by the AHS $\mathrm{E} 2 \mathrm{H}$ project. The intervention and control hospitals were selected based on comparable characteristics: annual number of ED visits and patient acuity according to the Canadian Triage and Acuity Scale (CTAS) [11]. Figure 1 shows the study flow diagram and lists specific hospital sites. Interventions at the study hospitals were variable, and are detailed in Table 1.

\section{Study population}

Seniors aged $\geq 65$ with an ICD-10 discharge diagnosis of fall, fracture, sprain, strain, laceration, contusion, superficial injury, or bursitis were included for possible analysis. Patients with a discharge diagnosis of hip fracture or trimalleolar ankle fracture were excluded due to their $100 \%$ need for operative intervention. Patients who had presented to an Alberta ED for a MSK complaint within the previous 30 days were also excluded.

Overall, 919 patients seen by the EDCC during their ED visit were matched with patients who had not been seen by the EDCC. Of this group, 9 pairs were excluded from the study: 2 pairs were excluded because outcome data was not available for one member of each pair and 7 pairs were excluded because one member of each pair 


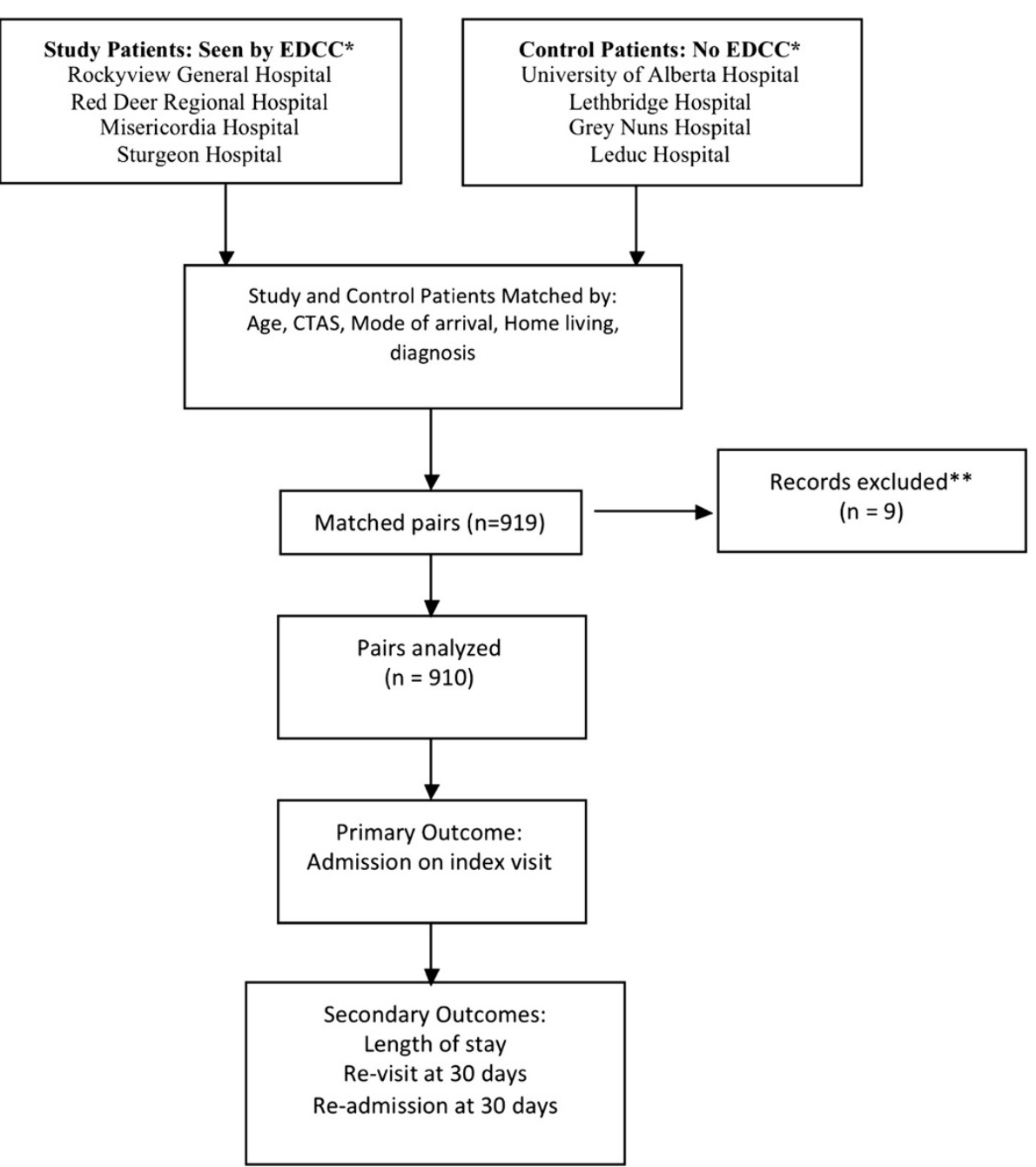

Figure 1 Study flow. *Data Sources: E2H Clerical Staff and DIMR (Data Integration, Measurement and Reporting). **Exclusions: Missing outcome data or left ED without being seen.

Table 1 E2H pilot project sites and main project activities

\begin{tabular}{ll}
\hline Site & Main project activities \\
\hline $\begin{array}{l}\text { Misericordia Community } \\
\text { Hospital }\end{array}$ & Introduced new EDCC role \\
$\begin{array}{ll}\text { Sturgeon Community } \\
\text { Hospital }\end{array}$ & Introduced new EDCC role \\
$\begin{array}{l}\text { Red Deer Regional } \\
\text { Hospital }\end{array}$ & $\begin{array}{l}\text { Increased EDCC hours by providing an } \\
\text { additional 1.4 EDCC full time equivalents for } \\
\text { evening and weekend coverage, introduced } \\
\text { clerical support for the EDCC, expanded home } \\
\text { care coverage, and enhanced the role of the }\end{array}$ \\
& $\begin{array}{l}\text { ED pharmacist and physiotherapist. } \\
\text { Introduced clerical support for Transition }\end{array}$ \\
& $\begin{array}{l}\text { Coordinators. The EDCC role was already } \\
\text { Rockyview General } \\
\text { Hospital }\end{array}$ \\
& $\begin{array}{l}\text { support was need thed to support the } \\
\text { volume of referrals. }\end{array}$
\end{tabular}

left the emergency room before assessment could be completed. The analysis dataset consisted of 910 matched pairs.

In the analysis dataset, most pairs (832) were matched on age (plus or minus 3 years), CTAS, mode of arrival (ambulance or walk-in), and living arrangements (home or institution) (Table 2). Of these, 231 pairs were also matched on diagnosis type (patients were matched by above criteria, but also had identical discharge diagnosis, e.g., Colles fracture, rib contusion); 45 pairs were matched on age, CTAS, and mode of arrival only; 25 pairs were matched on only age and CTAS; and 8 pairs were not CTAS matched, but differed by one. As all matches were made from like-hospitals, all pairs were also matched on hospital type. The criteria used to match pairs are shown in Table 2. Of the 910 pairs, 188 (20.7\%) were matched on age, CTAS, mode of arrival, and living arrangements and had identical MSK diagnoses; 44 pairs (4.8\%) were matched on age, CTAS, mode of arrival, living arrangements, and 
Table 2 Matched pairs by matching criteria

\begin{tabular}{|c|c|c|c|c|c|c|}
\hline \# Matched pairs $(n=910)$ & Age $( \pm 3 \mathrm{yr})$ & CTAS score & Mode of arrival $^{1}$ & Living arrangements $^{1}$ & Discharge diagnosis & Hospital type \\
\hline 601 & $x$ & $x$ & $x$ & $x$ & & $x$ \\
\hline 231 & $x$ & $x$ & $x$ & $x$ & $x$ & $x$ \\
\hline 45 & $x$ & $x$ & $x$ & & & $x$ \\
\hline 25 & $x$ & $x$ & & & & \\
\hline 8 & $x$ & & $x$ & $x$ & & $x$ \\
\hline
\end{tabular}

${ }^{1} \mathrm{AHS}$ is the single health authority responsible for delivering health care in the province of Alberta, Canada.

had the diagnosis of "tendency to fall - not elsewhere classified (NEC)". Matching was performed by a trained data abstractor who was blinded to the outcome data.

\section{Analysis}

A matched analysis was performed on the study sample, using McNemar's test for dichotomous outcomes (admission at baseline, revisit within 30 days, and admission within 30 days after baseline). A paired $t$-test was used to compare LOS between EDCC patients and controls. The analysis was performed on the entire study population and then on subgroups of patients with identical MSK diagnoses and "tendency to fall - NEC" diagnoses. The identical MSK diagnoses subgroup compared patients with the exact same discharge diagnosis, in an effort to control for comparison of MSK injuries of potentially differing severity. The tendency to fall NEC group represented patients who did not have an MSK diagnosis, but an underlying co-morbidity (e.g., urinary tract infection, exacerbation of chronic disease) that led to their ED presentation.

The data was provided by DIMR in Microsoft Excel (2007) files and then imported to Stata Software: Release 12 for statistical analysis [12].

\section{Results}

Baseline characteristics of the study population are shown in Table 3.

Of the 1,820 patients included in the study (EDDC and non-EDCC), 547 (30.1\%) were admitted to hospital at the baseline and 354 (19.5\%) revisited emergency during the 30-day follow-up period; 140 patients (7.7\%) were admitted to hospital within the follow-up period.

Our study was designed with $90 \%$ power to detect a difference of $6 \%$ between discordant pairs, assuming 989 pairs and approximately $20 \%$ discordant pairs [13]. However, our sample of 910 pairs had $15.6 \%$ in EDCC admit/ non-EDCC non-admit, and 13.7\% EDCC non-admit/ non-EDCC admit, a difference of only $1.9 \%$. As a result, our sample of 910 had only $18.5 \%$ power to detect this small $1.9 \%$ difference. However, a $1.9 \%$ difference, while statistically significant, may not be clinically significant.

Comparison of baseline admission rates found no statistically significant differences between EDCC patients and non-EDCC patients in the odds of baseline admission, revisit at 30 days or readmission at 30 days:

- Admission rates $(\mathrm{OR}=0.88 ; 95 \% \mathrm{CI}, 0.69$ to 1.12$)$

- Revisit rates at 30 days (OR = 1.19; 95\% CI, 0.95 to 1.51 )

- Readmission rates at 30 days $(\mathrm{OR}=1.03$; 95\% CI, 0.73 to 1.46$)$.

LOS data on initial hospital admission was available for 93 patients where both EDCC patients and controls were admitted. Mean LOS for EDCC patients was 8.15 days longer than mean LOS for controls $(P=0.065)$, a non-significant difference. Looking only at those with MSK diagnoses where both EDCC patients and controls were admitted $(n=14)$, mean LOS for EDCC patients was 6.2 days shorter than controls $(P=0.34)$. Looking only at those with "tendency to fall - NEC" diagnoses where both EDCC patients and controls were admitted $(n=20)$, mean LOS for EDCC patients was 7.4 days shorter than controls $(P=0.33)$. None of these differences are statistically significant.

Table 4 shows dichotomous outcomes for the entire sample. Tables 5 and 6 show subgroup analysis where diagnoses are MSK and "tendency to fall - NEC". Results are similar to the whole group analyses.

\section{Discussion}

This retrospective matched study examined the impact of an ED-based intervention for geriatric patients in the

Table 3 Baseline characteristics

\begin{tabular}{lcc}
\hline & $\begin{array}{c}\text { Non-EDCC patients, } \\
\mathbf{n = 9 1 0}\end{array}$ & $\begin{array}{c}\text { EDCC patients, } \\
\mathbf{n}=\mathbf{9 1 0}\end{array}$ \\
\hline Age, years (Std. dev) & $80.5(8.0)$ & $80.5(8.0)$ \\
Female, number (\%) & $623(68.5)$ & $643(70.7)$ \\
CTAS $=2$, number (\%) & $69(7.6)$ & $69(7.6)$ \\
CTAS $=3$ & $575(63.2)$ & $578(63.5)$ \\
CTAS $=4$ & $257(28.2)$ & $253(27.8)$ \\
CTAS $=5$ & $9(1.0)$ & $10(1.1)$ \\
Arrival by ground ambulance, & $518(56.9)$ & $541(59.5)$ \\
number (\%) & & $194(21.3)$ \\
Lives in institution, number (\%) & $147(16.2)$ & \\
\hline
\end{tabular}


Table 4 Dichotomous outcomes: whole sample

\begin{tabular}{lcccccc}
\hline & $\begin{array}{c}\text { Frequency of } \\
\text { outcome } \\
\text { number, (\%) } \\
\mathbf{n}=\mathbf{1 8 2 0}\end{array}$ & $\begin{array}{c}\text { Number of } \\
\text { EDDC patients } \\
\text { with outcome } \\
(\%) \mathbf{n}=\mathbf{9 1 0}\end{array}$ & $\begin{array}{c}\text { Number of } \\
\text { non-EDCC } \\
\text { patients with } \\
\text { outcome (\%) } \mathbf{n}=\mathbf{9 1 0}\end{array}$ & $\begin{array}{c}\text { Number (\%) of pairs where } \\
\text { EDCC experienced the } \\
\text { outcome and non-EDCC } \\
\text { did } \mathbf{n o t} \mathbf{n}=\mathbf{9 1 0}\end{array}$ & $\begin{array}{c}\text { Number (\%) of pairs where } \\
\text { non-EDCC experienced the } \\
\text { outcome and EDCC } \\
\text { did } \mathbf{n o t}, \mathbf{n}=\mathbf{9 1 0}\end{array}$ & $\begin{array}{c}\text { McNemar's } \\
\text { test: } \mathbf{p} \text {-value }\end{array}$ \\
\hline Admissions & $547(30.1)$ & $282(31.0)$ & $265(29.1)$ & $142(15.6)$ & $125(13.7)$ \\
Revisits & $354(19.5)$ & $164(18.0)$ & $190(20.9)$ & $132(14.5)$ & $158(17.4)$ & 0.3275 \\
Readmission & $140(7.7)$ & $69(7.6)$ & $71(7.8)$ & $63(6.9)$ & $65(7.1)$ & 0.1420 \\
\hline
\end{tabular}

form of assessment by an EDCC. This study found no statistically significant differences in the hospital admission rates, LOS or ED re-visits within 30 days of an initial visit for seniors seen by an EDCC and those not seen by an EDCC.

There are several possible explanations for the outcomes of patients included in this study. Selection bias in the sample of EDCC-assessed seniors used in the study may have affected the admission rate on the index visit. Seniors visiting an ED are not randomly selected for assessment by the EDCC; rather, the EDCC likely identifies seniors at risk of falling in the community who would be otherwise discharged from the ED. By bringing these patients to the attention of the ED staff and having them admitted, this may offset some of the admissions the EDCCs are themselves preventing. Further, many of the patients being seen by the EDCC are frail and present with multiple co-morbidities. Many of these patients may have been admitted regardless of EDCC involvement. Unfortunately, because of the retrospective study design, screening tools that could potentially identify patients at higher risk of readmission were not used for patient selection [14].

An important goal in the care of seniors is to deliver targeted multidisciplinary care to achieve improved health outcomes [8]. However, the impact of ED-based interventions in reducing elderly hospitalization and recidivism has been variable [4]. In the DEED II study, comprehensive geriatric assessment of ED patients aged 75 and older showed a lower rate of hospital admissions at 30 days and a reduction in ED admissions at 18 months [8]. The 30 day re-visit and re-hospitalization rates in DEED II were $22 \%$ and $16 \%$, respectively, compared with $19.5 \%$ and $7.7 \%$ in our study [8]. However, the DEED II study population differed in that all patients assessed were discharged from the ED on their initial visit, and followed-up with a CGA. Thus, it is difficult to compare this with the patients in our study. Furthermore, our study targeted seniors with MSK diagnoses, and thus we may expect a smaller impact than that seen in DEED II. CGA for discharged ED patients was also performed in a study by Mion et al., in this case seniors aged 65 and older were assessed by an advance practice nurse and referred to appropriate outpatient services [15]. This study showed a reduction in nursing home admissions at 30 days, but no reductions in repeat ED visits or hospitalizations. Guttman et al., evaluated the impact of a nurse discharge plan coordinator in a Canadian setting, a role similar to an EDCC, on unscheduled seniors ED revisits at 8 and 14 days [9]. Although a modest positive effect was shown in reducing unscheduled revisits, again this study population consisted exclusively of patients that were discharged from the ED. In a study by Gagnon et al., nurse case management of elderly patients discharge from the ED resulted in higher rates of ED readmission, with no improvement in other outcomes [16]. In all of these studies, seniors were discharged from the ED on the index visit, and all enrolled patients were community-dwelling and not institutionalized.

In our study, all seniors aged $\geq 65$ could potentially be seen and interviewed by the EDCC, including many in whom hospital admission would likely be unavoidable. However, some seniors were not seen by the EDCC due to extremes of acuity or already supportive living situations. For example, a trauma patient requiring intensive care unit admission or an ill medical patient would not be candidates for EDCC intervention, as there was no possibility of imminent discharge. Similarly, a patient with minor complaints and excellent home supports (e.g., children and spouse providing care) would not be seen by the EDCC. Therefore, a sensitivity analysis could not be performed to compare patients seen by the EDCC who were

Table 5 Dichotomous outcomes: patients with primary MSK diagnosis

\begin{tabular}{|c|c|c|c|c|c|c|}
\hline & $\begin{array}{c}\text { Frequency of } \\
\text { outcome } \\
\text { number, (\%) } \\
n=376\end{array}$ & $\begin{array}{l}\text { Number of } \\
\text { EDCC patients } \\
\text { with outcome } \\
(\%) n=188\end{array}$ & $\begin{array}{c}\text { Number of } \\
\text { non-EDCC } \\
\text { patients with } \\
\text { outcome }(\%) n=188\end{array}$ & $\begin{array}{l}\text { Number (\%) of pairs where } \\
\text { EDCC experienced the } \\
\text { outcome and non-EDCC } \\
\text { did not, } n=188\end{array}$ & $\begin{array}{l}\text { Number (\%) of pairs where } \\
\text { non-EDCC experienced } \\
\text { the outcome and EDCC } \\
\text { did not, } n=188\end{array}$ & $\begin{array}{l}\text { McNemar's } \\
\text { test: } p \text {-value }\end{array}$ \\
\hline Admissions & $84(22.3)$ & $48(25.5)$ & $36(19.1)$ & 25 (13.3) & $13(6.9)$ & 0.0730 \\
\hline Revisits & $76(20.2)$ & $34(18.1)$ & $42(22.3)$ & 25 (13.3) & $33(17.6)$ & 0.3581 \\
\hline Readmission & $28(7.4)$ & $13(6.9)$ & $15(8.0)$ & $12(6.4)$ & $14(7.5)$ & 0.8450 \\
\hline
\end{tabular}


Table 6 Dichotomous outcomes: patients with primary diagnosis of "tendency to fall"

\begin{tabular}{lcccccc}
\hline & $\begin{array}{c}\text { Frequency of } \\
\text { outcome } \\
\text { number, (\%) } \\
\mathbf{n}=\mathbf{8 8}\end{array}$ & $\begin{array}{c}\text { Number of } \\
\text { EDCC patients } \\
\text { with outcome } \\
(\%) \mathbf{n}=\mathbf{4 4}\end{array}$ & $\begin{array}{c}\text { Number of } \\
\text { non-EDCC } \\
\text { patients with } \\
\text { outcome (\%) } \mathbf{n}=\mathbf{4 4}\end{array}$ & $\begin{array}{c}\text { Number (\%) of pairs where } \\
\text { EDCC experienced the } \\
\text { outcome and non-EDCC } \\
\text { did } \mathbf{n o t} \mathbf{n}=\mathbf{4 4}\end{array}$ & $\begin{array}{c}\text { Number (\%) of pairs where } \\
\text { non-EDCC experienced the } \\
\text { outcome and EDCC did } \\
\text { not, } \mathbf{n}=\mathbf{4 4}\end{array}$ & $\begin{array}{c}\text { McNemar's } \\
\text { test: } \mathbf{p} \text {-value }\end{array}$ \\
\hline Admissions & $68(77.3)$ & $36(81.2)$ & $32(72.3)$ & $12(27.3)$ & $5(18.2)$ & 0.5034 \\
Revisits & $12(13.6)$ & $6(13.6)$ & $6(13.6)$ & $5(11.4)$ & $3(6.8)$ & $11.4)$ \\
Readmission & $6(6.8)$ & $4(9.1)$ & $2(4.6)$ & $(2.3)$ & 1.000 \\
\hline
\end{tabular}

not eligible for EDCC intervention. Based on the baseline characteristics of the participants, the study patients were of moderate to high acuity, with $70 \%$ being CTAS 2 or 3, and $57 \%$ to $60 \%$ arriving by ambulance. The average age of $80.5 \pm 8.0$ and large percentage of institutionalized patients also indicates this study's patients were of higher than average risk. While no statistically significant outcomes were identified in this study, several trends were noted. Whole group revisits at 30 days trended toward a reduction for patients who were seen by an EDCC when compared with the no EDCC group $(P=0.14)$. In the subgroup of patients with identical MSK diagnosis, there was a trend toward increased admission on index visit in the study group, when compared with the controls $(P=0.07)$. Finally, in the LOS evaluation, the mean LOS for the EDCC patients was 8.15 days longer than controls with a trend toward statistical significance $(P=0.065)$.

\section{Conclusions}

This study showed no reduction in baseline admission rate, revisits at 30 days, readmissions at 30 days, or hospital LOS for seniors aged $\geq 65$ seen by an EDCC, when compared with similar seniors not seen by an EDCC. However, this study had several limitations that may have affected the results, including the study design, sample bias, and variable nature of interventions at different hospital sites. There may be additional benefits of EDCC intervention, such as improved patient satisfaction, quality of life, and a reduction in functional decline. In the future, we would suggest further evaluation of other benefits provided by the EDCC role, as well as longer-term evaluation of in-patient admission and ED utilization rates at 6 months or 1 year.

Potential limitations of this study were numerous, and included study design, heterogeneity of interventions, and chosen outcomes. First, a retrospective matched study design is inferior to a prospective, randomized study design. Without a prospective design, patient selection and screening is not possible, and from previous studies of geriatric interventions, selection of an appropriate intervention population is paramount [4]. Our population was heterogeneous in both the type and severity of their discharge diagnoses, and while representative of a typical ED population, some of these patients may not be appropriate for EDCC intervention. Second, the nature of the EDCC intervention varied among hospital sites, in that the program was implemented from scratch at two sites and expanded upon at the other two sites. Third, it has been difficult to show the positive impact of an ED intervention on the outcomes chosen in this study, as has been documented in multiple previous studies [4]. Other outcomes, such as reduction in functional decline and quality of life improvement, may be more appropriate proxies for quality improvement in this domain. Fourth, the administrative database did not record patient function (e.g., activities of daily living), mobility, and comorbidities. Matching using these additional criteria would strengthen the overall conclusions and their absence had potential to confound the results. Fifth, the positive downstream effects of the EDCC on recidivism may not be apparent using a 30 day cutoff. Perhaps a window of 6 months or longer is more appropriate for analysis of these outcomes.

\section{Endnote}

${ }^{\mathrm{a}} \mathrm{AHS}$ is the single health authority responsible for delivering health care in the province of Alberta, Canada.

\section{Abbreviations}

AHS: Alberta Health Services; CGA: Comprehensive geriatric assessments; CTAS: Canadian Triage and Acuity Scale; DIMR: Data Integration, Measurement and Reporting; ED: Emergency department; EDCC: Emergency Department Care Coordinator; E2H: Emergency to Home Project; LOS: Length of stay; MSK: Musculoskeletal; NEC: Not elsewhere classified.

\section{Competing interests}

The authors declare that they have no competing interests.

Authors' contributions

$E A F, K A A, D W$, and $V L$ performed statistical analysis of the data. CA, JD, VL, and AT gathered the data, performed data linkage and created the dashboards for analysis. AT, JD, and QC were responsible for the Emergency to Home Project leadership and oversight. $C M B, E L, A T, J D, V L$, and $Q C$ conceived the study design. CMB drafted the manuscript, all other authors contributed to revisions for the final submission. All authors read and approved the final manuscript.

\section{Author details}

${ }^{1}$ Department of Emergency Medicine, Foothills Medical Centre, Room C231, 1403-29 St NW, Calgary, Alberta T2N 2 T9, Canada. ${ }^{2}$ Department of Family Medicine, University of Calgary, 2500 University Drive NW, Calgary, Alberta T2N 1 N4, Canada. ${ }^{3}$ Alberta Health Services, Seventh Street Plaza, 10030-107 St NW, Edmonton, Alberta T5J 3E4, Canada. ${ }^{4}$ Alberta Health Services, Reporting Services, Data Integration Measurement and Reporting, NWII, Suite 300, 4520-16 Ave NW, Calgary, Alberta T3B 0 M6, Canada. 


\section{References}

1. Aminzadeh F, Dalziel WB: Older adults in the emergency department: a systematic review of patterns of use, adverse outcomes, and effectiveness of interventions. Ann Emerg Med 2002, 39:238-247.

2. McKevitt EC, Calvert E, Ng A, Simons RK, Kirkpatrick AW, Appleton L, Brown DR: Geriatric trauma: resource use and patient outcomes. Can J Surg 2003, 46(3):211-215.

3. McCusker J, Verdon J: Do geriatric interventions reduce emergency department visits? J Gerontol 2006, 61A:53-62.

4. Hastings $\mathrm{SN}$, Heflin MT: A systematic review of interventions to improve outcomes for elders discharged from the emergency department. Acad Emerg Med 2005, 12(10):978-986.

5. Basic D, Conforti D, Rowland J: Standardised assessment of older patients by a nurse in an emergency department. Aust Health Rev 2002, 25:50-58.

6. Gold S, Bergman H: A geriatric consultation team in the emergency department. J Am Geriatr Soc 1997, 45:764-767.

7. Poncia HD, Ryan J, Carver M: Next day telephone follow up of the elderly: a needs assessment and critical incident monitoring tool for the accident and emergency department. J Accid Emerg Med 2000, 17:337-340.

8. Caplan GA, Williams AJ, Daly BD, Abraham K: A randomized controlled trial of comprehensive geriatric assessment and multidisciplinary intervention after discharge of elderly from the emergency department - the DEED II study. J Am Geriatr Soc 2004, 52:1417-1423.

9. Guttman A, Afilalo M, Guttman R, Colacone A, Robitaille C, Lang E, Rosenthal S: An emergency department-based discharge coordinator for elder patients: does it make a difference? Acad Emerg Med 2004, 11:1318-1327.

10. Alberta Innovates Health Solutions: ARECCI Ethics Screening Tool. http://www. ahfmr.ab.ca/arecci/screening.

11. Beveridge R: The Canadian Triage and Acuity Scale: a new and critical element in health care reform. J Emerg Med 1998, 16:507-511.

12. StataCorp: Stata Statistical Software: Release 12. College Station, TX StataCorp LP; 2011.

13. Connor RJ: Sample size for testing differences in proportions for pairedsample design. Biometrics 1987, 43:207-211.

14. Moons P, De Ridder K, Geyskens K, Sabbe M, Braes T, Flamaing J, Milisen K: Screening for risk of readmission of patients aged 65 years and above after discharge from the emergency department: predictive value of four instruments. Eur J Emerg Med 2007, 14:315-323.

15. Mion LC, Palmer RM, Meldon SW, Bass DM, Singer ME, Payne SM, Lewicki L, Drew BL, Connor JT, Campbell JW, Emerman C: Case finding and referral model for emergency department elders: a randomized clinical trial. Ann Emerg Med 2003, 41:57-68.

16. Gagnon AJ, Schein C, McVey L, Bergman H: Randomized controlled trial of nurse case management of frail older people. J Am Geriatr Soc 1999, 47:1118-1124.

doi:10.1186/1865-1380-7-18

Cite this article as: Bond et al:: The emergency to home project: impact of an emergency department care coordinator on hospital admission and emergency department utilization among seniors. International Journal of Emergency Medicine 2014 7:18.

\section{Submit your manuscript to a SpringerOpen ${ }^{\circ}$ journal and benefit from:}

- Convenient online submission

- Rigorous peer review

- Immediate publication on acceptance

- Open access: articles freely available online

- High visibility within the field

- Retaining the copyright to your article

Submit your next manuscript at $\gg$ springeropen.com 